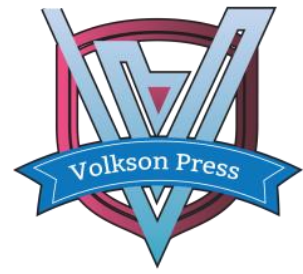

Contents List available at VOLKSON PRESS

Economics \& Management Innovations(EMI)

DOI : http://doi.org/10.26480/icemi.01.2017.384.388

\title{
Identification of occupational accident causes by use the Ishikawa diagram and Pareto principles
}

\author{
Adam Górny \\ Poznan University of Technology, Faculty of Management Engineering, \\ 11 Strzelecka St, room 318/2, PL-60-965 Poznań, Poland \\ e-mail: adam.gorny@put.poznan.pl \\ This is an open access article distributed under the Creative Commons Attribution License, which permits unrestricted use, distribution, and reproduction in any \\ medium, provided the original work is properly cited.
}

\section{ARTICLE DETAILS}

\section{Article History:}

Received 02 october 2017

Accepted 06 october 2017

Available online 11 october 2017

\section{Keywords:}

Occupational accident, Causes of

accident, Ishikawa diagram,

Pareto
principles..

\section{ABSTRACT}

Accidents trigger a number of responses, among them searches for their primary and secondary causes and for solutions expected to eliminate irregularities. According to Polish legislation, the duty to respond in such ways rests with the employer. In order to fulfil their duties and respond appropriately, persons responsible for dealing with accidents need tools which will help them perform their tasks. One available option is the Ishikawa diagram which helps identify accident causes and the primary causes of events. The paper describes how the Ishikawa diagram can be applied to analyse the overturning of a forklift truck. The Showed that Pareto principles, used to supplement the Ishikawa diagram, makes it possible to identify key causes of accidents in areas in need of immediate improvement.

\section{Introduction}

Occupational accidents are a major economic problem. They generate costs which are incurred by and affect the entire enterprise. Poland is the site of ten thousands accidents every year. Their victims include ca. 500 fatalities and a much greater number of injuries [1-2]. The number of accidents, the resulting damage and the relevant legislation require action to ensure that accidents are properly assessed. A study on accident causes should shed light on the root causes of accidents and suggest appropriate corrective and preventive measures so as to eliminate the most probable causes [3].

Every accident gives rise to damage, losses or a combination of both. Losses pertain to property damage while damage may also refer to people [4-6]. Damage may be bodily or psychological, including post-traumatic distress $[7,8]$.

The paper proposes to identify causes of occupational accidents by means of the Ishikawa diagram which is a traditional quality-oriented tool for verifying events whose consequences run contrary to expectations. According to the author, the tool can be used to search for and classify accidents. It offers a simple method of identifying accident causes which contribute to the greatest accident-related losses.

\section{Occupational accidents}

\subsection{The nature of occupational accidents and its causes}

The Polish law indirectly refers to a whole range of circumstances which meet the definition of occupational accident. Under the law, an occupational accident is a sudden occurrence brought on by an external cause which results in injury or death and occurs in the course of or in relation to the performance of work.

The primary goal in analysing an accident is to identify its causes and the circumstances in which the occurrence has taken place. The circumstances surrounding an accident are in fact of great importance. An objective picture of an accident can only be captured by running a systematic multidisciplinary study of the technical, psychological, organizational and managerial aspects of the occurrence. Another area of focus is an assessment of the psychological environment, traumas which may result from prior events and the time elapsed between the trauma and the accident itself [7-10]. All this is instrumental in identifying the true causes of accidents.

In the course of their work, employees are frequently exposed to factors which pose a hazard and work strain and are characteristic of specific workstations. In terms of their impact on the workforce, such factors can be classified as $[1,2,6,11]$ :

dangerous, i.e. factors whose impact results in or may lead to injuries, deleterious, i.e. factors whose impact causes or may cause illnesses,

strenuous, i.e. factors resulting in severe to excessive fatigue.

Depending on their severity and characteristics of the overall environment in which it occurs, a factor originally classified as strenuous may become deleterious while a deleterious factor may turn dangerous.

Regardless of their nature, all of the above factors may constitute a primary cause of an occupational accident. Accidents at work in Poland are caused mainly by improper $[1-3,12]$ :

worker behaviour (over 55\%), including workers caught off guard by an unexpected event, insufficient focus on an activity at hand disregard for hazards, ignorance of occupational safety laws and guidelines, self-willed behaviour, improper use of physical property, mental conditions contrary to occupational safety and failure to use protection items assigned to a workstation,

work and workstation organisation (approx. 27\%), including lacking or insufficient supervision over employees, supervisors tolerating breaches of safety rules, lacking or misguided occupational safety instructions, including manuals for the operation of machinery and equipment and the performance of technological processes, admission to work of untrained or insufficiently trained persons, failure to supply personal protection items to employees or selecting wrong protection items, improper coordination of collective work, insufficient occupational training, underqualified workforce, admission of employees with disqualifying medical conditions or without physical examinations,

condition of physical assets (approx. 11\%), including poor 
technological condition or poor or misguided selection of protection items (such as shields and covers barring access to dangerous zones), unstable physical assets (such as machinery not bolted down to floor, inappropriate positioning of the centre of gravity), lacking or improper collective protection measures and means, lacking or poorly selected control and alarm signalling devices or control and alarm signalling devices in poor technical condition, insufficiently durable physical assets, concealed flaws of physical assets, and

\section{use of tools and means of production (approx. 7\%).}

A study of the data provided in field literature shows that the severity of accident hazards is directly proportional to the intensity of the immediate factors which pose accident risks. When investigating the causes of an accident, it is essential to ascertain what has happened and why the events have unfolded despite all solutions and measures meant to mitigate accident risk. The commonly identified causes of accidents are [6]:

tolerating and ignoring hazards in the working environment,

human errors committed by employees,

reckless decision-making,

unreliable safety systems.

It is safe to assume that every accident results from the operation of either a single or multiple factors. Such factors are shortcomings and other irregularities which directly or indirectly contribute to the occurrence of an accident. The factors involve physical items (technical causes), the organization of work-flows in the workplace, workstation arrangements (organizational causes) and employee-related factors (human causes). The factors identified in the course of investigating accidents additionally include events (factors), which made directly or indirectly result in accident-related injuries and constitute indirect causes of accidents.

An investigation of the causes and impacts of accident occurrences requires an in-depth description and characterization.

\subsection{Occupational accident as a reason of hazard reduction}

The findings of such investigations are helpful in identifying areas of improvement and mitigating accident risks. As a consequence, measures are taken to eliminate hazards at source. These include the use of tools, equipment and facilities which ensure safety in the performance of work and keep hazards at bay. It is equally significant to design workstations ergonomically by ensuring that working conditions and work processes reflect employee abilities and, in particular, that individual workstations are properly organized, that work tools and furnishings are properly selected and, at the stage of design, work processes are made as unstrenuous as possible. Proper attention needs to be given to reducing the impact of individual factors by removing employees from their range of influence. This may apply to the use of automation and robots in the working environment as well as designing the work area to keep workstations as unaffected by hazards as possible. To protect employees to the required degree, employers need to reduce the impact of hazardous, deleterious and strenuous factors by adopting collective and individual protection measures. Collective measures should generally take priority over individual ones.

In order for any such measures to be effective, the persons involved must behave properly. It is in fact human behaviour that ultimately determines the effectiveness of any safety programmes. Such behaviours are motivated by the conviction of the concerned individuals that the measures are well-designed, adequately address the existing risk factors, can be applied in the specific production environment at hand and remain available in all external circumstances [9].

In selecting solutions designed to reduce accidents, significant focus is needed on the human factor. The subfactors at play include teaching employees to abide by rules of proper behaviour and the use of arrangements which increase the likelihood of such behaviour. It is essential to ensure that employees are dedicated and committed to approach their work positively. This, among other aspects, involves respect for the working rules and restrictions adopted for specific workstations $[3,8]$.

As a high proportion of occupational accidents are caused by humans, it is vital to improve ways in which workers are prepared for their work, inform them about hazards and risks, and make them adhere to workflow principles. The quality of the technical equipment used at the workplace is equally critical as it is vital for adapting workstations to match the psychophysical abilities of the worker [11, 13-16].

\section{Methodological assumptions for Ishikawa diagram and Pareto principles}

The Ishikawa diagram (i.e. the cause-and-effect diagram) is a popular tool for analysing cause-and-effect relationships. The tool's advantage lies in its capacity to present graphically relationships between causes which generate particular problems and analyse the existing relationships. Developed by Prof. Karou Ishikawa, the diagram was first used by Sumitomo Electric. It enables a broad analysis ranging from identifying accident impact to searching for all possible causes [3, 17-21]. The Ishikawa method may be used to analyse any events across a range of a company's activities. The method is perfectly suited for resolving problems which involve chains of multiple causes [18]. By ensuring the brainstorming team comprises representatives of various company functions, the organization makes it more likely that the causes of events will be presented objectively and comprehensively.

The five fundamental causes of irregularities are commonly referred to as the five M's - these stand for [17-19]:

Manpower: seen as the most complex and least predictable factor; the manpower factor covers skills and qualifications, habits, vocational experience, satisfaction levels and motivation,

Methods: seen as the most changeable and elusive of factors, methods cover all procedures and instructions followed in performing a task; methods include all modes of operation used in an organization and any relevant laws,

Machinery: a factor for analysing the equipment in use in terms of advancement, efficiency and safety as well as any license and certificates required for its application,

Materials: a factor used to analyse services and the processed materials,

Management: a factor involved in workflows and working conditions in an enterprise faced with a given problem; analysis of management extends to organizational structures, organizational cultures, shift systems, etc.

An equally common variant of the model is $5 \mathrm{M}+\mathrm{E}$ for environment. Each major cause incorporates component causes analysed individually and treated as problems to be solved.

The causes given above are those which occur most commonly. This depends on the type of activities the diagram is used to depict and the types of issues whose causes need to be investigated.

Secondary causes are closely related to the primary ones and, in fact, constitute their elaboration. While no stringent restrictions apply to the number of secondary causes, the transparency of the diagram requires it be kept down to a reasonable minimum and that tertiary factors be specified if and when needed. Once all factors which contribute to the end result to one degree or another have been identified, the most critical factor(s) may be selected. In choosing causes having the most impact on a given problem or aspect under consideration, use may be made of the Pareto analysis.

Pareto analysis relies on the empirically confirmed principle whereby 20 $30 \%$ of causes (factors) occurring in nature, technology and any other human activities commonly produce $70-80 \%$ of effects [21-24]. Put more generally, a small number of causes, persons or circumstances are responsible for the majority of occurrences.

The Ishikawa diagram helps to identify the causes of problems while the Pareto method allows one to arrange them by priority on the basis of the impact linked to a specific cause, as assessed by a team in charge of identifying the causes of irregularities. The team should therefore comprise individuals who have the required specialised knowledge and sufficient experience, are sufficiently objective, view the issues at hand from various perspectives and recognise the true purpose of their analysis $[25,26]$. Such team members must be willing to capture the causes of irregularities and have a proper understanding of the purpose of their assessment [12]. Their examination is to focus on the problem at hand and its causes rather than on identifying the culprits who have brought it about.

\section{Practical application of Ishikawa diagram and Pareto principles}

Efforts to improve the working environment and conditions tend to run into problems which may be difficult to identify unambiguously. To resolve the issue, it is necessary not only to gain awareness of the mechanisms behind a specific process but also to ascertain where the problem takes place and what causes have contributed to its occurrence. 
Once such knowledge has been acquired, it is possible to eliminate the problem and prevent any future recurrence.

At its core, the Ishikawa diagram offers a graphical presentation of various factors contributing to the overall final effect which helps assess their impacts. The model can be used to identify mutual links contributing to a specific problem. The Ishikawa diagram may be seen in a subject-based layout $[18,19]$. This is particularly desirable when the effects in question can be linked to technical or organizational ones and assigned to separate problem sets. In such a case, effects are marked on the main horizontal axis. The diagonal axes are used to mark problem areas while the horizontal ones which connect to the diagonal axes show causes directly affecting a given problem area.

The key to achieving the desired effect is to find the correct diagnosis and accurately and unambiguously recognise the causes of irregularities. The Ishikawa diagram technique relies on the knowledge of experts and regular employees who join forces to draw up the diagram. Once prepared, the diagram puts in order all knowledge on a specific well-defined issue in a clear structure. To succeed in reaching the desired conclusions, the composition of the assigned team needs to be well-balanced. It is therefore acceptable to select its members deliberately to ensure the accident will be described accurately. The main purpose of working team meetings is to identify the primary and secondary causes of events. The number of such meetings will thus be as required to provide an accurate description. It will depend on the ability of the team members to develop an accurate description of the accident in question. The essence, therefore, of this cause-effect diagram is to bring together any causes which may result in a specific effect so as to study how such factors evolve.

Success in achieving the expected result depends mainly on following prescribed methodologies by proceeding through a number of steps arranged in a specified sequence $[3,25,26]$ :

Step 1: analyse the issue at hand to identify the problem accurately and clearly; this helps identify the key categories of causes and link them with any problems one faces,

Step 2: draw the diagram; this requires breaking the causes down into main and subordinate components which affect the assessment of the main causes,

Step 3: check if your diagram is complete; this requires recognising and analysing the causes which were previously omitted in detailed analyses,

Step 4: analyse the diagram; this requires choosing a small number (typically 2 to 4 ) of causes having the greatest impact on the overall effect, analysing them and answering the question of whether the identified cause does indeed contribute to the problem at hand. It is essential to formulate the findings as conclusions with reference to any available corrective measures. Pareto analysis may be used in analysing the diagram.

When needed, the detailed causes may be expanded to detect any supplementary contributing factors. In plotting the Ishikawa chart, certain difficulties may be encountered $[3,17]$. One of them ties to the absence of any competences needed to associate information and differentiate between primary and secondary causes. The resulting diagram is then unclear and makes it impossible properly to examine and verify causes in terms of their significance. As a consequence, the user will be prevented from identifying and undertaking adequate corrective and preventive measures.

Another challenge faced in developing the Ishikawa diagram lies in allocating individual causes to proper sets. Usually, individual causes may be assigned to two or more sets. While assignment errors are not critical, they may stand in the way of formulating the right conclusions [19].

This diagram-based analysis relies, at its core, on following the above steps to identify the true causes of accidents and defining the scope and method for adopting modifications to prevent its recurrence. Experience shows it is best to get experts from various disciplines associated with a problem to draw the diagram together. Persons involved in analysing a given issue should be highly knowledgeable in their field, be motivated to reveal the causes of defects (rather than hiding the effects and true causes of errors) and have a good understanding of the purpose of the exercise $[3,17,18]$. The problem or process irregularity should be analysed until each cause can be assigned, with a sufficient degree of certainty, to a category of causes which either can or cannot be eliminated.

5. Causal analysis occupational accidents (for example of forklift truck driver)

\subsection{Characteristic of work [3]}

The object of detailed analysis was the operator of forklift truck. The specific job of an operator forms part of an internal transport system which in turn makes up a component of the overall production process. The aim of transport is to perform tasks involving the moving of raw materials, supplies and finished goods. Hence, transport responsibilities include the supply of raw and other materials to warehouses, their distribution to individual sections, the delivery of finished goods to warehouses, the carrying of finished goods to users, the moving of waste, etc.

Forklift operator drives an industrial vehicle equipped with load lifting devices and also does auxiliary activities related to job description. The operator's main task is to transport and move about loads inside the facility and store it in either low-bay or high-bay warehouse. The vehicle is steered by both levers and pedals. The drivers in question have been found to be exposed to excessive physical strain and forced to spend long durations in strained postures. This adversely affects their psychophysical fitness. Maintaining unchanged postures over prolonged periods and vibration exposure compromises their performance. Driver errors and incorrect vehicle operation pose potential hazards increasing the risk of occupational accidents.

\subsection{Description of accidents causes}

Based on the problem at hand, it was possible to identify the causes of an accident. The use of the Ishikawa diagram helped to link the causes of effects with specific primary factors (manpower, methods, machinery, materials, management and environment). Thus, the Ishikawa diagram helped to identify new causes and causes which need to be examined to produce a satisfactory assessment of the accident event. As a consequence, all of the information available has been ordered into a transparent and telling structure. The outcome can easily be used to spot issues to be addressed to eliminate accident causes.

The assessment team identified likely causes of an accident and matched them with factors shown in the Ishikawa diagram. They then analysed such causes and indicated the sub-causes contributing to the overall effect of fork-lift truck overturn. Such relationships are shown in Figure 1. The procedure made it possible to identify factors which trigger an accident and those which make its occurrence more likely.

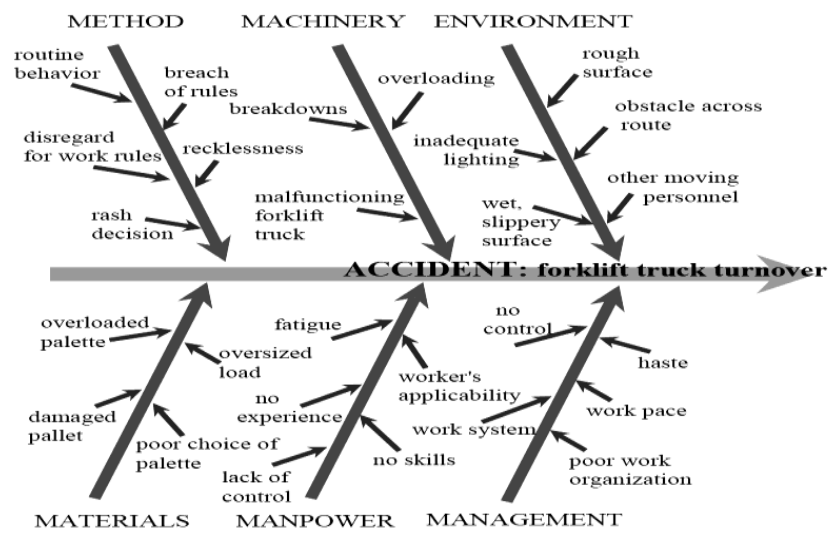

Fig. 1. Ishikawa diagram for identified occupational accidents causes

An examination of the diagram shown in Fig. 1 shows that the most common trigger of accidents is human error involving the use of a machine taking place in unfavourable workplace circumstances and hazards. The circumstances and hazards themselves, however, may not be treated as the sole cause of an accident [6]. Their role is limited to being parts of an environment which is more or less conducive to the occurrence of an accident. The actual accident needs a trigger in the form of an event in which an employee loses control over the situation.

One might assume that occupational accidents result not only from unfavourable working conditions found in the working environment but also from mistakes, mainly organizational, leading to high-risk behaviours [6]. In their prevention programs, therefore, each enterprise should expand their hazard recognition systems and place proper emphasis on proposing measures which mitigate the impact on employee health and life. 
The examination rested on the assumption that accidents are highly complex. An accident event results from a combination of a whole array of untoward technical, organizational, environmental and human factors. Such events are usually preceded by the occurrence of unsafe conditions in the working environment $[3,27,11,26,28]$. The order in which such accident-contributing factors arise may vary. Whatever combinations the factors fall into, their end result is always an accident.

The initial cause of an accident is a distortion in the existing system. The distortion transforms circumstances into the so called hard situation [5]. To continue their work and to remain in the working environment, employees need specific knowledge. Employees who do have specific knowledge and/or experience are likely to deal successfully with a given set of circumstances.

The employees will then eliminate the distortion bringing the difficult situation back to normal. Another option is employees adjusting to an existing hazard. Adjustment is often a routine response which does not contribute to safety [29]. Failure to remove an existing distortion makes it more likely for an accident hazard to recur in the future.

Finally, note needs to be taken of the third option in which employees fail to handle the distortion. They are then overwhelmed (as their capacities are insufficient relative to the severity of the distortion). They therefore poorly interpret the situation and take a misguided decision allowing the accident to take place.

One may assume, therefore, that the occurrence of an accident depends on circumstances classified into the five basic categories of factors, i.e. [26]:

(1) external factors affecting the working environment,

(2) the availability of proper technical equipment at the work station

(3) work station organisation,

(4) the professional competence of the workers,

(5) the psychological condition of the workers.

Each team member tasked with assessing an accident may base his/her assessment on 0 to 5 causes of his/her choice which corresponds to a 0 to $2 \%$ share of the impact of a given cause (assuming the team is made up of 10 members). In effect, a certain percentage of the impact of a given cause on the accident was ascribed to such cause.

From among them, the Pareto principle was applied to select the six key causes which are the most likely to occur. These are:

in the area of method: routine behaviour as well as disregard for work rules and breach of work rules,

in the area of manpower: no skills and no experience,

in the area of management: haste.

The percentages of the impact of accident causes on the final event are provided in Table 1.

An analysis of accident causes showed the need for solutions which will make the workers more aware of hazards in their work environment and, in particular, hazards related to their specific jobs. Such causes may be classified as organizational. Before such causes can be ruled out and preventive action can be taken, it is necessary to define the causes of a given situation. In practice, it is often insufficient to limit oneself to the identification of incident causes. Unless such causes can be properly arranged and structured, it is very difficult to pick out the ones of critical importance and define the relationships between them. To streamline the analysis of root causes, it is crucial to apply a method for establishing a hierarchy of causes contributing to the observed problems.

Table 1. Detailed analysis of occupational accidents causes.

\begin{tabular}{|l|c|l|c|}
\hline $\begin{array}{c}\text { Causes of } \\
\text { occupational } \\
\text { accident }\end{array}$ & $\begin{array}{c}\text { influence on } \\
\text { overall } \\
\text { effect [\%] }\end{array}$ & $\begin{array}{c}\text { Causes of } \\
\text { occupational } \\
\text { accident }\end{array}$ & $\begin{array}{c}\text { influence on } \\
\text { overall } \\
\text { effect [\%] [) }\end{array}$ \\
\hline Method: & $\mathbf{9 4}$ & $\begin{array}{l}\text { Manpower: } \\
\text { - fatigue, }\end{array}$ & 50 \\
- routine & $\mathbf{8 6}$ & - no experience, & $\mathbf{8 2}$ \\
behaviour, & $\mathbf{8 4}$ & - worker's & 52 \\
- disregard for & 70 & applicability to & $\mathbf{9 8}$ \\
work rules, & 74 & work, & \\
- breach of rules, & & - no skills & \\
- recklessness, & & & \\
- rash decision & & & \\
\hline
\end{tabular}

\begin{tabular}{|c|c|c|c|}
\hline $\begin{array}{l}\text { Machinery: } \\
\text { - breakdowns, } \\
\text { - malfunctioning } \\
\text { forklift truck, } \\
\text { - overloading }\end{array}$ & $\begin{array}{c}74 \\
72 \\
78\end{array}$ & $\begin{array}{l}\text { Management: } \\
\text { - haste, } \\
\text { - work pace, } \\
\text { - poor work } \\
\text { organization, } \\
\text { - no control, } \\
\text { - work system }\end{array}$ & $\begin{array}{l}\mathbf{9 6} \\
72 \\
62 \\
60 \\
52\end{array}$ \\
\hline $\begin{array}{l}\text { Materials: } \\
\text { - overloaded } \\
\text { palette, } \\
\text { - oversized load } \\
\text { - damaged pallet, } \\
\text { - poor choice of } \\
\text { palette, } \\
\text { - lack of control }\end{array}$ & $\begin{array}{l}60 \\
62 \\
72 \\
68 \\
80\end{array}$ & $\begin{array}{l}\text { Environment: } \\
\text { - rough surface, } \\
\text { - obstacle across } \\
\text { route, } \\
\text { - other moving } \\
\text { personnel, } \\
\text { - wet, slippery } \\
\text { surface, } \\
\text { - inadequate } \\
\text { lighting }\end{array}$ & $\begin{array}{l}50 \\
50 \\
60 \\
42 \\
30\end{array}$ \\
\hline
\end{tabular}

Detailed analyses of accident causes shows clearly how essential the human aspect is as a factor for their occurrence. The effectiveness of measures aimed at improving occupational safety depends on the competencies of persons performing tasks in a given organization. Their success in deploying measures designed to improve the status quo hinges on technical innovations in the organization of work and on improvements in organizational culture, particularly those pertaining to safety. The human factor is central for an organization's ability to modify the working environment, enhance work rules and boost the potential for success in its endeavours $[20,14]$. A superior culture of safety reduces accident rates, eliminates onerous working conditions and improves near miss statistics. It helps to promote safe behaviours among employees consequently boosting the overall value of an organization.

The results suggest that the level of safety (expressed as accident rates) depends on more than mere technical accessories and adjustments made to make conditions in the working environment reflect human abilities [6, 16]. Accident rates are driven not only by technological factors and the working environment (as significant as they are), but also, and primarily so, by employees, especially those tasked with the job of overseeing working conditions. The frequency of accidents caused by any specific cause shows that employees tend to take risks while failing to learn from past mistakes. This may be due to their insufficient education and training, their personality traits as well as the tolerance of such behaviour by supervisors and employers [1-3].

For accident prevention to work effectively, it is crucial to lay down legislation describing technical and educational safety standards and binding procedures. An informal culture of safety needs to evolve and modify employees' approach to risk taking, their patterns of behaviour in high-risk situations, and their appreciation of the value of human health and life. For accident policies to work successfully, cooperation is needed in the areas of technology and education as well as psychology to bring out people's inner motivations to act safely $[6,16]$. If, however, no significant changes take place after an accident to prevent the future recurrence of similar dangerous events, then the threat will continue leading to further accidents in similar circumstances which are due to similar reasons.

\section{Conclusions}

The goal in analysing accident rates is to chart out the course of action to ensure that accidents are effectively prevented and make employees aware of the areas and work situations in which significant risks arise. The accident cause analysis for a workplace should identify factors contributing to accidents, including the types of tasks performed, the technologies employed, the highest risk work stations and the accidenthazard minimizing measures to be taken by the employer.

In-depth analysis of the sequence of events leading to an occupational accident is crucial for identifying its causes. Worker safety and proper selection of protection items best suited for specific workstation hazards depend largely on how regularly accident causes are identified. Hence, strict health and safety standards are key for business managers and occupational health and safety officers.

Ishikawa diagram makes it possible to:

respond to particular events comprehensively,

orderly manage information flows, 
- keep descriptions simple and clear,

- prioritise causes,

- link events and problems and identify their locations,

- eliminate causes which contribute to specific problems.

The main benefit of the Ishikawa diagram based analysis is its potential to provide insights into accident causes. This helps identify ways to effectively prevent occupational accidents [3, 26]. Effective accident prevention depends on how well an organization manages to eliminate the circumstances and causes of accident risks and potential accident hazards.

Once an organization identifies its accident-related issues, it will be in a position to take appropriate action allowing its employees to perform their work in a safe environment. This will enable it to reduce operating expenses [6,29] while meeting its obligation to provide workers with safe and hygienic working conditions.

\section{References}

[1] Working Conditions in 2014. Statistical Information and Elaborations (GUS, Warsaw, 2015)

[2] Working Conditions in 2015. Statistical Information and Elaborations (GUS, Warsaw, 2016)

[3] A. Górny, The use of Ishikawa diagram in occupational accidents analysis, in: P.M. Arezes, et al. (eds.), Occupational Safety and Hygiene, SHO 2013, p. 162-163 (SPOSHO, Guimaraes, 2013)

[4] F.J.M. Jackson, E.D. Fonseca, F.P.A. Lima, F.J.C.M. Duarte, Organizational factors related to occupational accidents in construction. Work, 41(sup. 1), 4130-4136 (2012)

[5] C.C. Jacinto, G. Soares, T. Fialho, P. Antono, S.A. Silva, An overview of occupational accidents notification systems within the enlarged EU. Work: A J. of Prevention, Assessment and Rehabilitation, 39(4), 369-378 (2011)

[6] J. Stelman, (Ed.), Encyclopaedia of Occupational Health and Safety (ILO, Geneva, 2012)

[7] J. Sadłowska-Wrzesińska, A. Górny, A. Mościcka-Teske, The outcomes of shift working the context of psychosocial functioning - sex aspects, in: P.M. Arezes, ed al. (Eds.), Occupational Safety and Hygiene IV, p. 197-201 (Taylor and Francis Group, London, 2016)

[8] S. Pezaro, W.Clyne, E.A. Fulton, A systematic mixed-methods review of interventions, outcomes and experiences for midwives and student midwives in work-related psychological distress, Midwifery, 50, 163-173

[9] A. Górny, Man as internal customer for working environment improvements, Procedia Manufacturing, 3, 4700-4707 (2015)

[10] O. Nuttman-Shwartz, Post-Traumatic Stress in Social Work, in: J.D. Wright (Ed.), International Encyclopaedia of the Social \& Behavioral Sciences, p. 707-713 (Elsevier, 2015)

[11] A. Perez , D.R.E Gonzales, Human error reduction at work. in: P.P. Mondelo, W. Karwowski, et al. (Eds), Commitment towards prevention: a corporate responsibility, Proc. 6th International Conference on Occupational Risk Prevention), p. 858 (CD-ROM) (ORP, La Coruna, 2008)
[12] A. Górny, The use of working environment factors as criteria in assessing the capacity to carry out processes, MATEC Web of Conferences, 94, 04011 (2017)

[13] M. Hassall, T. Xiao, P. Sanderson, A. Neal, Human Factors and Ergonomics, in: J.D. Wright (Ed.), International Encyclopaedia of the Social \& Behavioral Sciences, p. 297-305 (Elsevier, 2015)

[14] A. Górny, The Elements of Work Environment in the Improvement Process of Quality Management System Structure, in: W. Karwowski., G. Salvendy (Eds.), Advances in Human Factors, Ergonomics, and Safety in Manufacturing and Service Industries, p. 599-606 (CRC Press, Boca Raton, 2011)

[15] R. Mitchell, R. Friswell, L. Mooren, Initial development of a practical safety audit tool to assess fleet safety management practices, Accident Analysis \& Prevention, 47, 102-118 (2012)

[16] M.V. Stringfellow, Accident analysis and hazard analysis for human and organizational factors (Massachusetts Institute of Technology; Massachusetts, 2010) K. Ishikawa, Guide to Quality Control (Asian Productivity Organization, Tokyo, 1976)

[18] K. Ishikawa, J.H. Loftus (Eds.), Introduction to quality control. (3A Corporation, Tokyo, 1990)

[19] K. Ishikawa, What is Total Quality Control? The Japanese Way (New Jersey, Prentice Hall, 1985)

[20] Ch-T. Su, Quality Engineering (CRC Press, Boca Raton, 2013)

[21] I. Kaliszewski, Quantitative Pareto Analysis by Cone Separation Technique (Kluwer Academic Pub., 1994)

[22] A. Górny, Identification of accidents causes by the Pareto principle, in: P.M. Arezes, et al. (Eds.), Occupational Safety and Hygiene, SHO 2015, p. 143-145 (SPOSHO, Guimaraes, 2015)

[23] K.M. Vijay, How Well Do We Know Pareto Optimality?, J. of Economic Education, 22(2), 172-178 (1991)

[24] A. Certa, G. Galante, T. Lupo, G. Passannanti, Determination of Pareto frontier in multi-objective maintenance optimization, Reliability Engineering \& System Safety, 96(7), 861-867 (2011)

[25] A. Hamrol, Zarządzanie jakością. Teoria i praktyka (Quality management. Theory and practice) (PWN, Warsaw, 2008), In Polish

[26] E. Kindlarski E, Wykresy Ishikawy i Pareto (Ishikawa and Pareto diagrams) (Bellona, Warsaw, 1993), In Polish

[27] A. Misztal, M. Butlewski, Live improvement at work (Publishing House of Poznań University of Technology, Poznań, 2012)

[28] J.G. Backhaus, J.A.H. Maks (Eds.), From Walras to Pareto (Springer; Berlin Heidelberg:, 2006)

[29] A. Górny, Ergonomics in occupational safety formation ergonomic requirements in system management of industrial safety, Foundations of Control and Management Sciences,11, 127-137 (2008) 